Brigitte Holzinger \& Eirin Fränkl

Le rêve lucide comme technique de psychothérapie
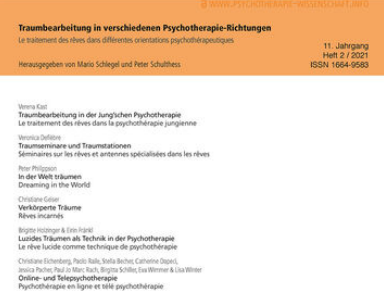

\section{Psychotherapie-Wissenschaft}

11. Jahrgang, Nr. 2, 2021, Seite 65-66

DOI: 10.30820/1664-9583-2021-2-65

Psychosozial-Verlag 


\section{Impressum}

Psychotherapie-Wissenschaft

ISSN 1664-9583 (Print-Version)

ISSN 1664-9591 (digitale Version)

11. Jahrgang Heft $2 / 2021$

https://doi.org/10.30820/1664-9583-2021-2

info@psychotherapie-wissenschaft.info

www.psychotherapie-wissenschaft.info

\section{Herausgeber}

Schweizer Charta für Psychotherapie in der Assoziation

Schweizer Psychotherapeutinnen und Psychotherapeuten

Geschäftsstelle ASP

Riedtlistr. 8

CH-8006 Zürich

Tel. +41432689300

www.psychotherapie.ch

\section{Redaktion}

Mara Foppoli, Lugano

Lea-Sophie Richter, Zürich

Mario Schlegel, Zürich

Peter Schulthess, Zürich

Hinweise für AutorInnen befinden sich auf der Homepage der Zeitschrift: www.psychotherapie-wissenschaft.info

\section{Verlag}

Psychosozial-Verlag

Walltorstr. 10

D-35390 Gießen

+49642196997826

info@psychosozial-verlag.de

www.psychosozial-verlag.de

\author{
Abo-Verwaltung \\ Psychosozial-Verlag \\ bestellung@psychosozial-verlag.de
}

\section{Bezugsgebühren}

Jahresabonnement 44,90€ (zzgl. Versand)

Einzelheft $24,90 €$ (zzgl. Versand)

Studierende erhalten gegen Nachweis $25 \%$ Rabatt.

Das Abonnement verlängert sich um jeweils ein Jahr, sofern nicht eine Abbestellung bis acht Wochen vor Ende des Bezugszeitraums erfolgt.

ASP-Mitglieder wenden sich wegen des Abonnements bitte direkt an die ASP.

\section{Anzeigen}

Anfragen zu Anzeigen bitte an den Verlag:

anzeigen@psychosozial-verlag.de

Es gelten die Preise der auf www.psychosozial-verlag.de einsehbaren Mediadaten.

ASP-Mitglieder wenden sich bitte direkt an

die ASP-Geschäftsstelle: asp@psychotherapie.ch

\section{Digitale Version}

Die Zeitschrift Psychotherapie-Wissenschaft ist auch online einsehbar: www.psychotherapie-wissenschaft.info

\section{(C) $(1) \Theta$}

Die Beiträge dieser Zeitschrift sind unter der Creative Commons Attribution-NonCommercial-NoDerivs 3.0 DE Lizenz lizensiert. Diese Lizenz erlaubt die private Nutzung und unveränderte Weitergabe, verbietet jedoch die Bearbeitung und kommerzielle Nutzung. Weitere Informationen finden Sie unter: creativecommons.org/licenses/by-nc-nd/3.0/de 


\title{
Le rêve lucide comme technique de psychothérapie
}

\author{
Brigitte Holzinger \& Eirin Fränkl \\ Psychotherapie-Wissenschaft 11 (2) 2021 65-66 \\ www.psychotherapie-wissenschaft.info \\ CC BY-NC-ND \\ https://doi.org/10.30820/1664-9583-2021-2-65
}

Mots clés : rêve lucide, rêve clair, formation sur le rêve lucide, cauchemar, psychothérapie

Le rêve lucide est un phénomène de rêve particulier qui intègre des aspects de la conscience et du sommeil en une seule expérience. Il se caractérise avant tout par la conscience qu'a la personne qui rêve qu'elle est en train de rêver. Dans les rêves lucides, les personnes qui rêvent sont capables d'intervenir activement dans l'événement du rêve et de le modifier (LaBerge, 1980 ; LaBerge \& Rheingold, 1991). Les critères suivants de Holzinger (2014) servent à définir le terme de rêve lucide :

1. Conscience de l'orientation (spatiale)

2. Conscience de la capacité de choisir

3. Conscience d'être dans un état de concentration (intense) (conscience du «flux », voir Csikszentmihalyi et al., 2014)

4. Conscience de l'identité (le «moi »)

5. Conscience de l'environnement du rêve

6. Conscience de la signification et du contenu du rêve

7. Conscience de la mémoire

Les critères 1 et 2 sont essentiels pour l'expérience d'un rêve lucide et doivent tous deux être remplis pour qu'un rêve soit considéré comme lucide. Ils permettent de classer le rêve dans la catégorie des rêves lucides et de le distinguer des phénomènes connexes tels que le rêve prélucide et la paralysie du sommeil.

Ces dernières années, le rêve lucide a suscité un intérêt croissant en tant que sujet de recherche, notamment en neuropsychologie. Cependant, peu de recherches ont été menées sur l'utilisation du rêve lucide comme approche thérapeutique en psychothérapie. La formation sur les rêves lucides (FRL) représente une approche thérapeutique innovante et très prometteuse. La FRL comprend des éléments de psychoéducation, des exercices de relaxation, la tenue d'un journal du sommeil et des rêves et l'enseignement des techniques de rêve lucide. Dans le traitement des cauchemars, notamment dans le cadre du syndrome de stress post-traumatique, les premiers résultats prometteurs en termes d'efficacité ont déjà été enregistrés.

L'effet du rêve lucide sur les cauchemars est principalement attribué au fait que l'auto-efficacité perçue (Lancee et al., 2010), la responsabilité personnelle et le contrôle de soi sont renforcés par la FRL. En outre, le rêve lucide permet la confrontation avec des stimuli anxiogènes, ce qui peut réduire la peur des images de cauchemar (Holzinger, 2014). Le rêve lucide a également été associé à la santé mentale en dehors du cadre thérapeutique, ainsi qu'à une meilleure estime de soi (Doll et al., 2009), à une perception accrue de la maîtrise de soi et de la créativité (Hess et al., 2017) et à une résilience psychologique accrue face à des situations de stress et de menace (Soffer-Dudek et al., 2011).

Si le rêve lucide peut avoir de nombreuses possibilités et des effets positifs, il doit être utilisé avec prudence chez certains groupes de personnes. Son utilisation dans la psychose est déconseillée car le rêve lucide peut potentiellement exacerber les hallucinations et le délire, favorisant ainsi le mélange de la réalité interne et externe chez les patients/tes psychotiques (Mota et al., 2016). En général, les patients/tes ayant des tendances à perdre le sens de la réalité peuvent ne pas être adaptés au rêve lucide (Holzinger, 2014).

Malgré des résultats prometteurs, la recherche sur le rêve lucide en tant qu'approche thérapeutique en psychothérapie en est encore à ses débuts. Des recherches supplémentaires dans ce domaine sont nécessaires pour mieux comprendre les mécanismes du rêve lucide en psychothérapie et pour développer la FRL en conséquence.

\section{Les auteures}

Dr. Brigitte Holzinger exerce en tant que psychothérapeute pour la psychothérapie comportementale intégrative dans son propre cabinet. En tant que directrice de l'Institut de recherche sur la conscience et le rêve à Vienne, elle mène actuellement des projets de recherche sur les thèmes du rêve, du rêve lucide, des troubles du sommeil et de la qualité du sommeil. Elle dirige également un cours de certificat postuniversitaire sur le coaching du sommeil à la Faculté de médecine de Vienne. Vous trouverez de plus amples informations sur les cours en ligne et sur DreamSenseMemory à l'adresse https://www.traum.ac.at.

Eirin Fränkl, BSc, fait des études de psychologie, mention psychologie clinique, et de psychologie de la santé dans le cadre du programme de maîtrise de l'Université de Vienne. Elle travaille comme assistante de recherche à l'Institut de recherche sur la conscience et le rêve. 
Traumbearbeitung ... | Le traitement des rêves ...

\section{Contact}

Dr. Brigitte Holzinger

Canongasse 13/1

1180 Wien

E-Mail : brigitte.holzinger@meduniwien.ac.at 